\title{
Bloch Surface Waves Based Platform for Integrated Optics
}

\author{
R.Dubey*, E. Barakat and H. P. Herzig \\ Optics \& Photonics Technology Laboratory (OPT), \\ École Polytechnique Fédérale de Lausanne (EPFL), \\ Rue de la Manadière 71b, CH-2002 Neuchâtel 2, Switzerland \\ email:* richa.dubey@epfl.ch
}

\begin{abstract}
A dielectric multilayer platform sustaining Bloch surface waves is investigated for planar integrated optics. We study the optical properties of high refractive index material $\left(\mathrm{TiO}_{2}\right)$ and obtain propagation lengths in the range of millimeters.

Keywords-Bloch surface waves (BSW), Multilayer platform, Scanning near-field optical microscopy (SNOM), Two-dimensional optics, Photonics, Optics, Light and matter interaction, Micro and nanostructures.
\end{abstract}

\section{INTRODUCTION}

Optical surface electromagnetic modes, highly confined to the interface, which can be excited in the photonic band gap of truncated dielectric periodic multilayers are called Bloch surface waves (BSW). Dielectric materials possess low loss characteristics which results in high propagation length and large resonance strength. These features are highly desirable for two dimensional (2D) optics and hence make BSW as a best candidate for integrated optics domain [1]. Another advantage is, a strong field intensity, increased by several orders of magnitudes, can be achieved by tuning the maximum intensity associated with the BSW on the surface. Thereby enhance the light-matter interaction close to the surface. Furthermore, platform provides the possibility to work with both polarizations and with any wavelength by properly choosing the refractive index and thickness of the layers constituting the multilayer. Taking the advantage of field confinement, the platform has also an application in the sensing domain [2].

\section{PlatFORM CONCEPT AND METHODS}

The design of the multilayer platform is based on one dimensional photonics crystal which consists of six periodic stacks of alternative silicon dioxide $\left(\mathrm{SiO}_{2}\right)$ and silicon nitride $\left(\mathrm{Si}_{3} \mathrm{~N}_{4}\right)$ layers. The multilayer platform is fabricated using plasma-enhanced chemical vapor deposition (PECVD) and designed to work around the telecommunication wavelengths, $1.5 \mu \mathrm{m}$. The thicknesses of $\mathrm{SiO}_{2}$ and $\mathrm{Si}_{3} \mathrm{~N}_{4}$ layers are $472 \mathrm{~nm}$ and $283 \mathrm{~nm}$, and refractive indexes are 1.45 and 1.79 at $\lambda=$ $1500 \mathrm{~nm}$, respectively. Total thickness of the multilayer platform is around $4.5 \mu \mathrm{m}$. The whole pattern is deposited on a glass wafer just as a Bragg mirror shown in Fig.1. To terminate the periodicity of multilayers, a $50 \mathrm{~nm}$ thick layer of $\mathrm{Si}_{3} \mathrm{~N}_{4}$, called top layer, is deposited on the top of the platform. The high index material, Titanium dioxide $\left(\mathrm{TiO}_{2}, \mathrm{n}=2.23\right)$, layer is deposited on the top of the platform (Bragg mirror + top layer) and will be used to shape the photonic devices on the top.

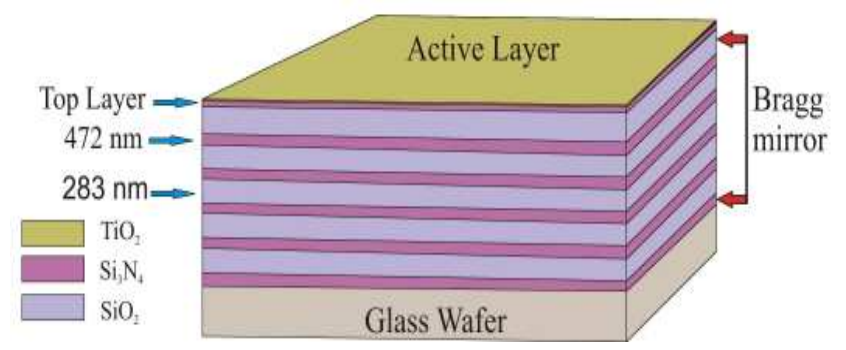

Fig.1 Schematic of BSW Multilayer platform (BSW-ML) deposited on the glass wafer.

To excite BSW, the propagation constant of the incoming beam should match with the propagation constant of BSW. Therefore, we use total internal reflection configuration for this purpose by using a BK7-glass prism. The schematic of the excitation configuration is shown in Fig. 2.

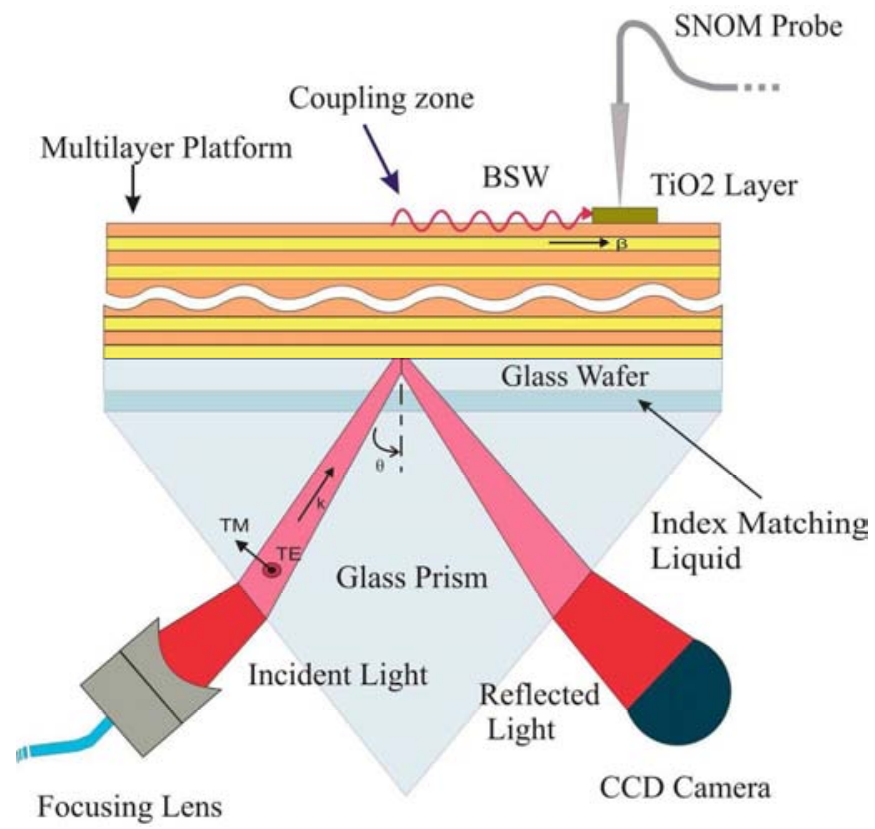

Fig.2. Total internal reflection configuration using BK7-glass prism to excite BSW. 
Because of the surface behavior of BSW, near field optical microscopy is the best tool for optical characterization. The evanescent surface waves is collected with a subwavelength aperture probe of multi-heterodyne scanning near-field optical microscope (MH-SNOM) which allows a simultaneous measurement of the amplitude and the phase is used for the optical.

\section{Result AND Discussion - PROPAGATION LENGTH AND EFFECTIVE REFRACTIVE INDEX ANALYSIS}

In this paper, we study two of the key parameters of BSW, such as, propagation length $\left(\mathrm{L}_{\mathrm{BSw}}\right)$ and the effective refractive index $\left(\mathrm{n}_{\text {eff }}\right)$. These parameters play an important role in characterizing the losses associated with the multilayer platform and determining the optical properties of 2D components.

The propagation length for bare multilayer and different thicknesses of $\mathrm{TiO}_{2}$ has been measured experimentally. The Near field image of BSW propagation has been shown in Fig.3. (a). $\mathrm{L}_{\mathrm{BSw}}$ is obtained by exponentially fitting the decrease of the field amplitude of the surface wave along the propagation direction, as demonstrated in Fig.3.(b) . For $15 \mathrm{~nm}$ thickness of $\mathrm{TiO}_{2}$, we achieved a propagation length of around 2.5 millimeters in near field, which is $\sim 30$ times longer than the recently obtained "Long-Range SPPs" studied by Lin et al [3] and $\sim 4$ times longer than the one studied by $\mathrm{L}$. $\mathrm{Yu}$ [4].

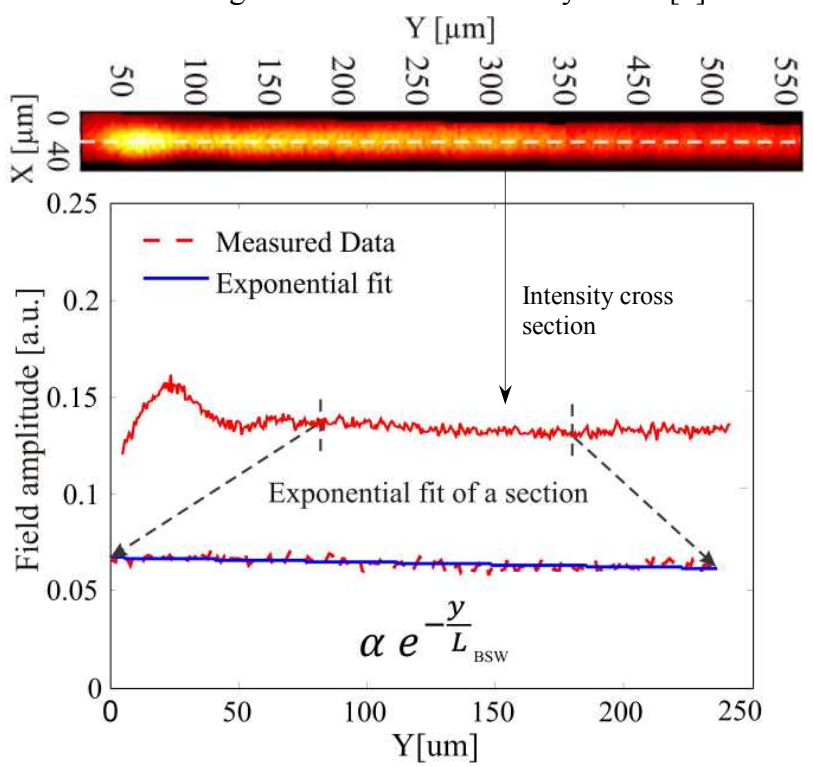

Fig.3.(a). Intensity distribution of $15 \mathrm{~nm}$ thick $\mathrm{TiO}_{2}$ layer, measured in near field, demonstrating BSW propagation. (b). Exponential fit of the field amplitude along the direction of propagation to measure propagation length.

Effective refractive index $\left(\mathrm{n}_{\text {eff }}\right)$ is introduced by propagation constant of surface waves. The presence of the additional layer modifies the local $n_{\text {eff }}$ which creates a contrast between bare multilayer and coated multilayer. The refractive index contrast $(\Delta \mathrm{n})$ between the platform and polymer layer affects the capability to manipulate BSW most effectively. We measured the refractive index contrast for different thicknesses of $\mathrm{TiO}_{2}$ such as $15 \mathrm{~nm}, 30 \mathrm{~nm}$ and $60 \mathrm{~nm}$. As the thickness of $\mathrm{TiO}_{2}$ increases, $\Delta \mathrm{n}$ shifts towards higher values.
In near field, $\Delta \mathrm{n}$ of 0.15 has been obtained for $60 \mathrm{~nm}$ thickness of $\mathrm{TiO}_{2}$. From the theory (matrix transfer method), it has been proven that $\Delta \mathrm{n}$ of $\sim 0.2$ can be achieved with $100 \mathrm{~nm}$ thickness of $\mathrm{TiO}_{2}$, which is good for low loss and compact photonic devices. In figure 4 , we plotted the theoretical and the measured results of $\Delta \mathrm{n}$ as a function of the $\mathrm{TiO}_{2}$ thicknesses. It can be seen that both results are close to each other. The effective index contrast has been confirmed in the near field and far field as well.

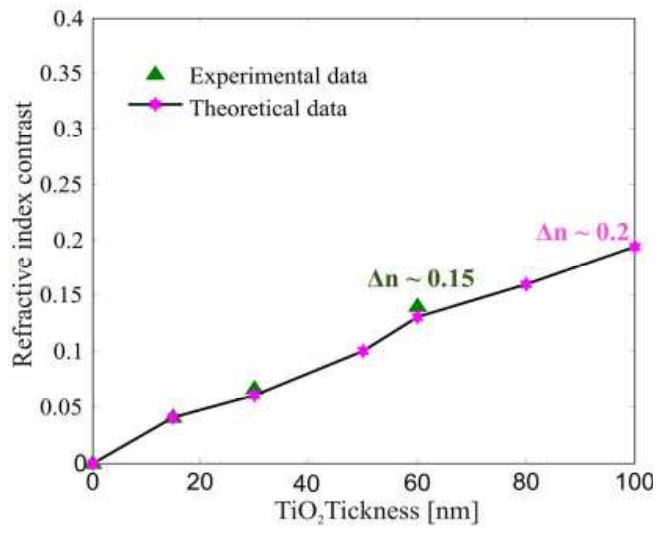

Fig.4. Plot of simulated and measured values of $\Delta \mathrm{n}$ for different thicknesses of $\mathrm{TiO}_{2}$

\section{CONCLUSION}

To conclude, dielectric multilayers are considered as a platform for a planner manipulation of the Bloch surface waves. We obtained a propagation length of $\sim 2.5$ millimeters for $15 \mathrm{~nm}$ thickness of $\mathrm{TiO}_{2}$ layer and a $\Delta \mathrm{n}$ of $\sim 0.15$ for $60 \mathrm{~nm}$ of $\mathrm{TiO}_{2}$. Experimental results show good agreement with simulation results.

Graphene layer and Silicon, as a high index material, are under near field investigation. In near future, we aim to characterize different optical components on the top of platform with the aid of multi-heterodyne scanning near-field optical microscopy in near field in near-infrared. These components include 2D Ring resonators and 2D Interferometers.

\section{ACKNOWLEDGMENT}

We acknowledge funding from Swiss National Science Foundation under the project number 200020135455 . We work in collaboration with Dr. Matthieu Roussey from the University of Eastern Finland for atomic layer deposition of $\mathrm{TiO}_{2}$ on the top of platform.

\section{REFERENCES}

[1] T. Sfez, et al.,"Two-Dimensional Optics on Silicon Nitride Multilayer: Refraction of Bloch Surface Waves.”, Applied Physics Letters , vol. 96, pp. 151101-1-3, 2010.

[2] Santi, S., et al., "Real-time Amyloid Aggregation Monitoring with a Photonic Crystal-based Approach.", ChemPhysChem, vol 14, pp. 3476-3482, Sepetember 2013.

[3] J. Lin, et al., "Cosine-Gauss Plasmon Beam: A Localized Long-Range Nondiffracting Surface Wave.”, Physical Review Letters, vol. 109, pp 093904, August 2012.

[4] L. Yu, et al., "Manipulating Bloch surface waves in 2D: a platform concept-based flat lens.", Light: Science \& Applications, vol. 3, January 2014 